\title{
UTILISING TIA AND ACUTE NEUROLOGY SERVICES TO THE MAXIMUM EFFICIENCY
}

\author{
Rebecca WHIliams, Kuven Moodley, Bhavini Patel \\ Department of Neurology, St George's University Hospitals NHS Foundation Trust
}

\section{TIA Services}

Transient ischaemic attack (TIA) clinics are a well-established part of stroke services. It has been observed that approximately $60 \%$ of all patients referred to the TIA service at St George's Hospital do not have a final diagnosis of TIA. With the emergence of acute neurology services, it would be beneficial to combine the strengths of both services to improve the percentages of positive TIA diagnosis in the TIA clinic in order to help the most patients. In August 2016, St George's Hospital started a 'Hot Clinic' (a rapid access neurology service) for acute neurological symptoms that are not thought to be TIA or seizures. In this clinic, all patients are seen within $\mathbf{5}$ days of referral.

\section{Objective}

To assess the effect of starting the Hot Clinic on the TIA service.

\section{Method}

Data from TIA clinics between March 2016 (6 months before the Hot Clinic started) and November 2017 were analysed. We compared the number of referrals, percentage of $\mathrm{TIA} /$ stroke/appropriate patients and percentage of neurological diagnoses before and after the acute neurology clinic started in August 2016.

\section{Results}

Between March 2016 and August 2016, we saw 317 patients in the TIA service compared to 257 patients in the 6 months following the initiation of the acute neurology service. The average number of patients referred to TIA clinic decreased to $\mathbf{4 2}$ per month as opposed to 52 per month prior to August 2016.

Prior to August 2016, the mean percentage of appropriate referrals was $37 \%$. The mean percentage of appropriate referrals in the year following the introduction of the acute neurology service was $34.3 \%$.

The main differential before and after August 2016 was headache and migraine aura; the percentage of patients with this diagnosis only decreased by $1 \%$ after August 2016. Twelve per cent of patients were diagnosed with symptoms of uncertain aetiology and this did not change after the introduction of the Hot Clinic.

After the Hot Clinic initially started, there was a rise in appropriate referrals (40-41\% in September and October 2016 respectively). Following this, there appears to be a steady rise in appropriate referrals to the TIA service between February 2017 and May 2017, and again from August 2017 to October 2017.

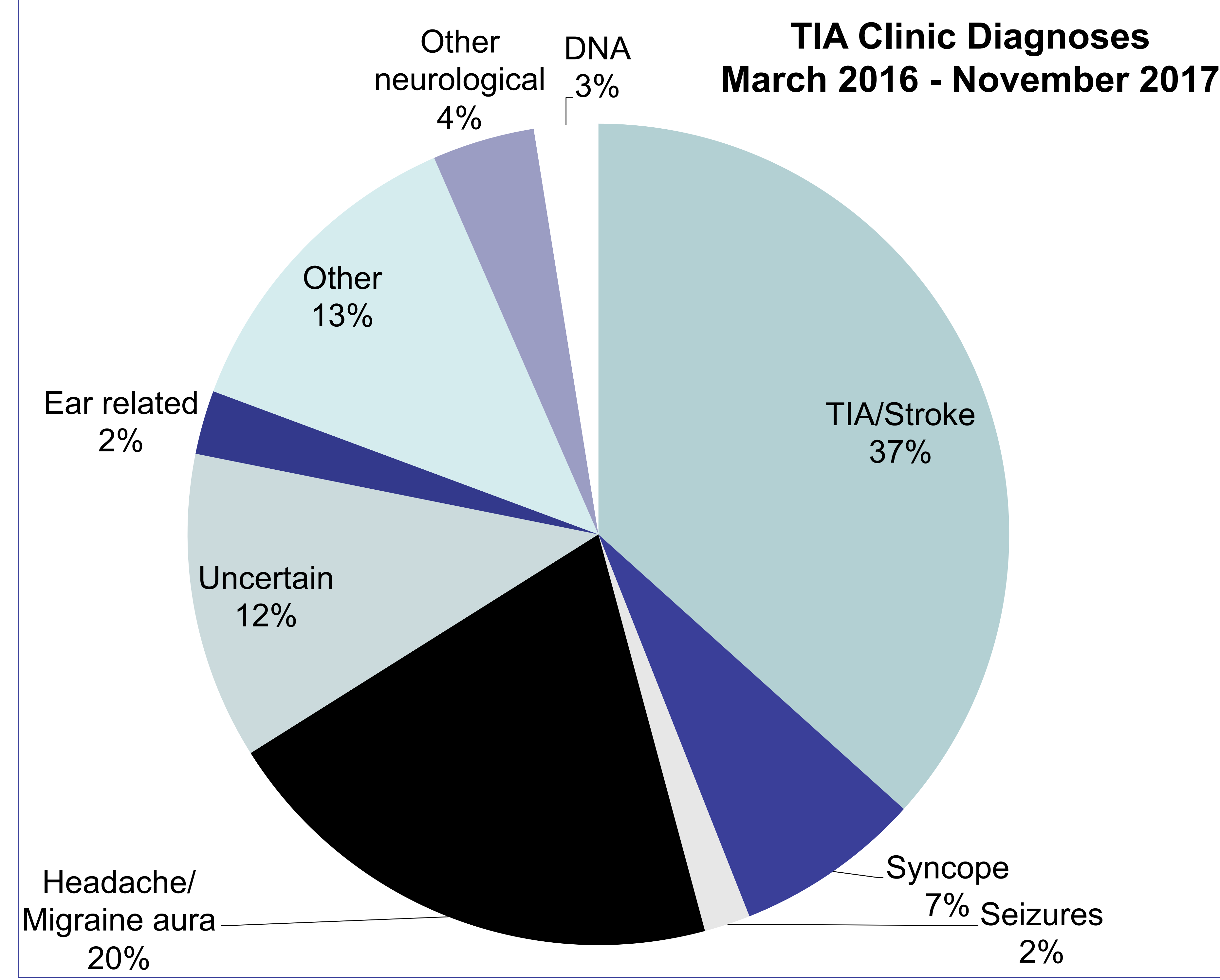

Figure 1: This pie chart demonstrates a breakdown of the diagnoses given in the TIA service between March 2016 to November 2017. The TIA/stroke section also includes amaurosis fugax and central retinal artery occlusion (CRAO). Some examples of 'other' diagnoses include musculoskeletal pain, delirium, stress, viral infections and medication side effects.

\section{Conclusion}

It is apparent that the introduction of the acute neurology service had some impact on the number of patients referred to TIA clinic initially. Now that this service is well-established it may be worth analyzing the data from 2018 to see whether the percentage of appropriate referrals has significantly increased. A well thought out model can be used to effectively use both TIA and acute neurology clinics to provide the best care for patients. It is clear that a large proportion of referrals to TIA clinic are actually migraine, therefore, providing education to GPs and acute medical staff regarding TIA diagnosis and how to differentiate this from migraine aura would reduce the burden on the TIA service. More education and thought is needed to ensure both services receive the correct type of referrals.

Percentage of Appropriate Referrals to TIA Clinic from September 2016 to November 2017

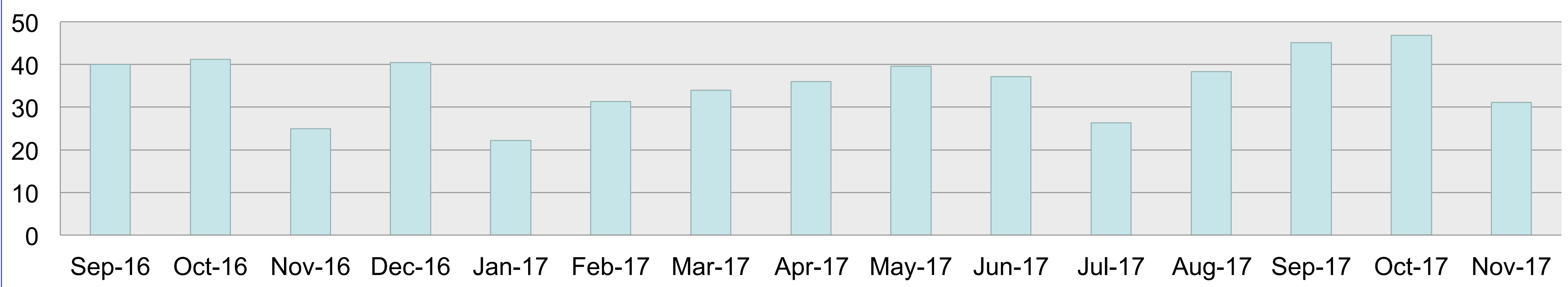

Figure 2: A bar chart showing the percentage of appropriate referrals (TIA, amaurosis fugax and CRAO) per month after the Hot Clinic was introduced.

Number of Patients Referred to the TIA Service and Proportion of Appropriate Referrals

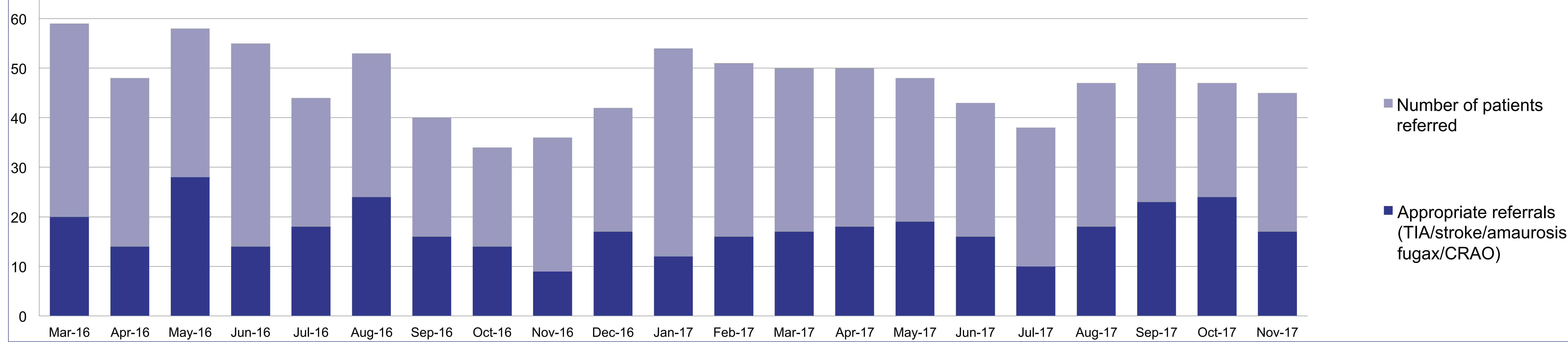

Figure 3: A bar chart comparing the number of patients seen in TIA clinic from March 2016 to November 2016 with the number of patients who were diagnosed as TIA/ stroke/amaurosis fugax/CRAO. 